\title{
A Qualitative analysis of the global earth model EIGEN-6C4 in predicting gravity disturbances over Goiás state, Midwest Brazil.
}

\author{
Allan Soares Ramalho ${ }^{1}$, Kristoffer A.T. Hallam ${ }^{2}$ and Rodrigo Bijani ${ }^{1}$ \\ 1 Universidade Federal Fluminense, Niterói, RJ \\ 2 Observatório Nacional, Rio de Janeiro, RJ
}

Copyright 2021, SBGf - Sociedade Brasileira de Geofísica.

This paper was prepared for presentation during the 17th International Congress of the Brazilian Geophysical Society, held in Rio de Janeiro, Brazil, 16 - 19 August, 2021.

Contents of this paper were reviewed by the Technical Committee of the 17th International Congress of The Brazilian Geophysical Society and do not necessarily represent any position of the SBGf, its officers or members. Electronic reproduction or storage of any part of this paper for commercial purposes without the written consent of the Brazilian Geophysical Society is prohibited.

\section{Abstract}

Gravity surveys are extremely important for applied geophysics, specially for evaluating and inferring relevant information about the density distribution of the Earth's interior. There are different gravity acquisition platforms for particular investigations. Depending on the accessibility, land measurements can really be prohibitive and ineffective, which may cause an insufficient spatial data coverage. Alternatively, aerial gravity measurements are important for improving the geophysical/geological investigation. However, the data obtained by this last type of acquisition may present undesirable noises due to oscillations and vibrations produced by the moving platforms. The use of Global Earth Models (GGMs) overcome these limiting aspects, once one is allowed to predict gravity anywhere at Earth's surface. This work presents a qualitative analysis based on the comparison of terrestrial and satellite gravity data. To obtain the gravity disturbances, one needs both the normal gravity and the geometric altitude. Once the gravity disturbances are computed for both data-sets, residuals, histograms and crossplots are presented to bespeak the relevant discrepancies. To validate the proposed method, a data-set covering Goiás state, Midwest Brazil is considered. All comparative analysis show, in an overall view, a reasonable resolution of GGM EIGEN-6C4 in modeling gravity and altimetric data, specially for greater wavelengths.

\section{Introduction}

The acquisition of gravity data is a valuable method in a large variety of geologic and geophysical studies. Once the gravity data is accurately acquired, one can infer the distribution of densities beneath the Earth's surface (Torge 1989). Over the decades, the acquisition of terrestrial gravity data become more efficient and precise, once new set of technological equipment were developed. Since 1978, the Observatório Nacional is the Brazilian institute responsible for the Rede gravimétrica fundamental brasileira (RGFB), by defining a set of gravity stations throughout the country (Escobar, 1987; Junior et al. 2019). Few time later, the transported gravimetry becomes also a very efficient mode of gravity acquisitions, once the amount of data could be dramatically improved and remote areas be easily accessed Halpenny and Darbha (1995); Bell et al. (1999). Around seventies, initial satellite missions also allow gravity data to be acquired abroad the Earth's surface, using complex accelerometer systems Schwintzer and Reigber (2002).

Terrestrial gravity provides a high-resolution data acquisition but measurements can really be prohibitive, depending on the area assessment. Airborne gravity can overcome such limitation, but the financial expenditures improve dramatically Halpenny and Darbha (1995). Satellite gravity data present even better global covering, but the data produced by shallow sources are not enough resolved in this data-set Barbosa (2017); Förste et al. (2016); Hallam (2019). The main goal of this work consists on a comparative analysis comprising observed and predicted gravity disturbances over Goiás state, Midwest Brazil. To do so, several terrestrial gravity stations all over the state are organized in a database and the gravity disturbance is then computed. After that, we use International Centre of Global Earth Models (ICGEM) website and also downloaded the absolute gravity from EIGEN-6c4 global earth model at the same stations. With this, we use the geometric altitudes of each station to compute normal gravity from Li and Götze (2001) and, consequently, obtain the predicted gravity disturbance. For a more consistent statistical comparison, crossplots and histograms are also provided and analyzed. Basically, the EIGEN-6c4 model is quite robust in predicting gravity and altimetric data, especially the latter. The gravity disturbance residuals presented in this study shows that the regional trend in the observed gravity data is well recovered, but the amplitudes are not, which is probably related to the spherical harmonics truncation of the model. As future investigations, other models would be considered into this methodology. Additionally, airborne gravity data could also be an interesting possibility for defining the wavelengths ranges modeled by global gravity models.

\section{Methods}

A main goal of this work lies in a comparison between gravity disturbances acquired from land surveys and the ones produced by EIGEN-6C4 (F'orste et al. 2014). To do so, Figure 1 presents the basis of this methodology. Basically, we need the gravity measurements and the geometric heights of a set of gravity stations. But before, we believe that some specific definitions of important gravity and altimetry elements are necessary. 


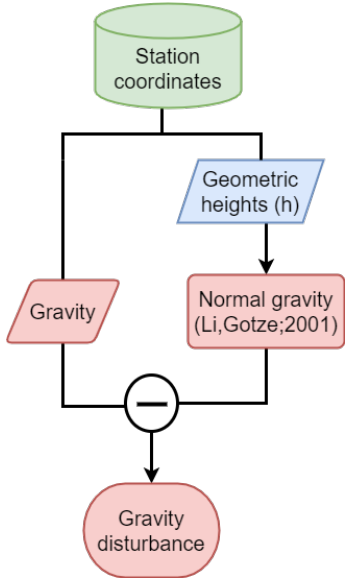

Figure 1: Flowchart of the main steps of the presented method.

\section{Real and normal gravity}

Imagine that $\mathbf{g}$ is the gravity vector, also named as real gravity, produced by the Real Earth, and can be defined as (Moritz, 1980, Hofmann-Wellenhof, 2005):

$$
\mathbf{g}=\nabla W_{\text {real }},
$$

in which $\nabla W_{\text {real }}$ is the gradient of the Gravitational and the centrifugal potential $W=V(\mathbf{r})+\phi(\mathbf{r})$ produced by a unit mass in a particular position on the Earth's surface $\mathbf{r}$. We can define a similar quantity, named normal gravity as:

$$
\gamma=\nabla W_{\text {normal }} .
$$

In this case, the gravitational potential is referred to the Normal Earth, which can be defined as the surface of an ellipsoid in revolution with the same angular velocity of the Earth Moritz (1980); Molina (2001); Escobar (2000). Figure 2 presents the major concerns on these particular surfaces.

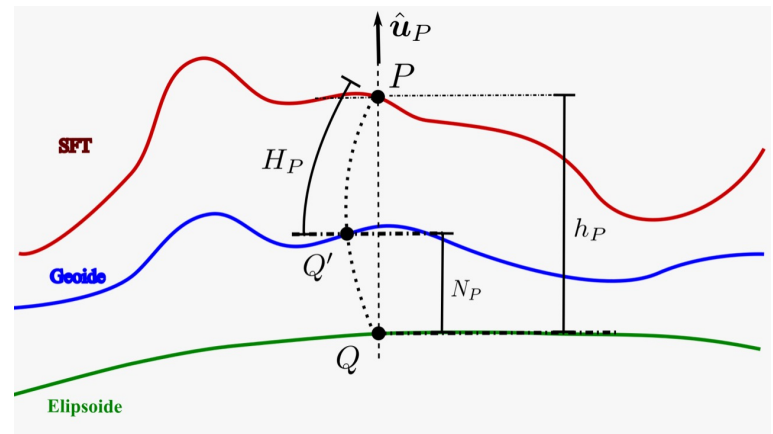

Figure 2: A 2D sketch of the three relevant surfaces and elements discussed in this work.Geometric height $\left(h_{p}\right)$ and Geoidal Undulation $\left(N_{p}\right)$.

\section{Gravity disturbance vector}

Imagine a particular point $P$ on the Earth's surface (Figure 2. A projection of this point over the Geoid and Normal
Earth are represented by $Q^{\prime}$ and $Q$, respectively. The gravity anomaly vector $\left(\delta g_{P}\right)$ can be defined as:

$$
\Delta \mathbf{g}_{\mathbf{P}}=\mathbf{g}_{\mathbf{Q}^{\prime}}-\gamma_{\mathbf{Q}}
$$

This means that difference between the real gravity at $Q^{\prime}$ and normal gravity at $Q$. We can naturally observe a lack of precision when Equation 3is defined, once two vectors with different projections are subtract. Alternatively, the gravity disturbance is then defined as:

$$
\delta \mathbf{g}_{\mathbf{P}}=\mathbf{g}_{\mathbf{P}}-\gamma_{\mathbf{P}}
$$

We now observe that Equation 4 computes the difference of gravity values over the same reference surface. This is a more rigorous definition that is going to be followed by us in this work. Additionally, to compute $\gamma_{\mathbf{P}}$, we use Li and Götze (2001).

\section{Geometric height}

To compute $\gamma_{\mathbf{P}}$ and $\gamma_{\mathbf{Q}}$ using Li and Götze (2001) we need the geometric heights. To obtain such measurements, geoid undulations and Normal Earth heights of the study area are required, once:

$$
h_{P} \approx H_{P}+N_{P} .
$$

Commonly, land surveys using Global Navigation Satellite System (GNNS) provide the geometric height $\left(h_{P}\right)$ without any further considerations. Commonly, some of these elements are not easy to compute. This is a natural reason for using models to predict some sort of dataset. With this concept in mind, we are ready to modeling gravity elements.

\section{Gravity Global Model: EINGEN-6C4}

We consider EIGEN-6C4 in this work due to a high-order degree expansion of the Spherical Harmonics(e.g., $n=$ 2190) Förste et al. (2006); Förste et al. (2016). This is an important aspect, once we are interested in modeling a broad range of wavelengths gravity data. This model offers a large variety of functional modes. In this work, we use both gravity_earth to compute the absolute gravity over the grid data points. Additionally, the functional gravity is also required when the user knows the Geodetic coordinates of each gravity station. For more details, the reader is invited to Barthelmes 2009,2014). Figure 3shows a general view of the website with all functionalities.

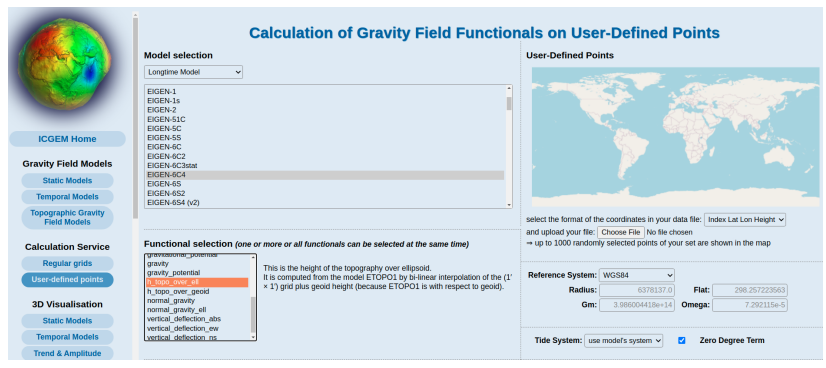

Figure 3: The website look of the International Centre for Global Earth Models (ICGEM). 


\section{Results}

\section{Geometric Heights (h)}

We start showing the comparison of geometric heights acquired throughout Goiás state, Midwest Brazil. In an overall view, both observed and modeled heights are quite similar, as can be seen in Figure 4 (a) and (b). The residuals presented in Figure 4 (c) reinforces the good quality of the modeled heights from EIGEN-6C4,once of the residual height values are mostly null (i.e., yellow colored).
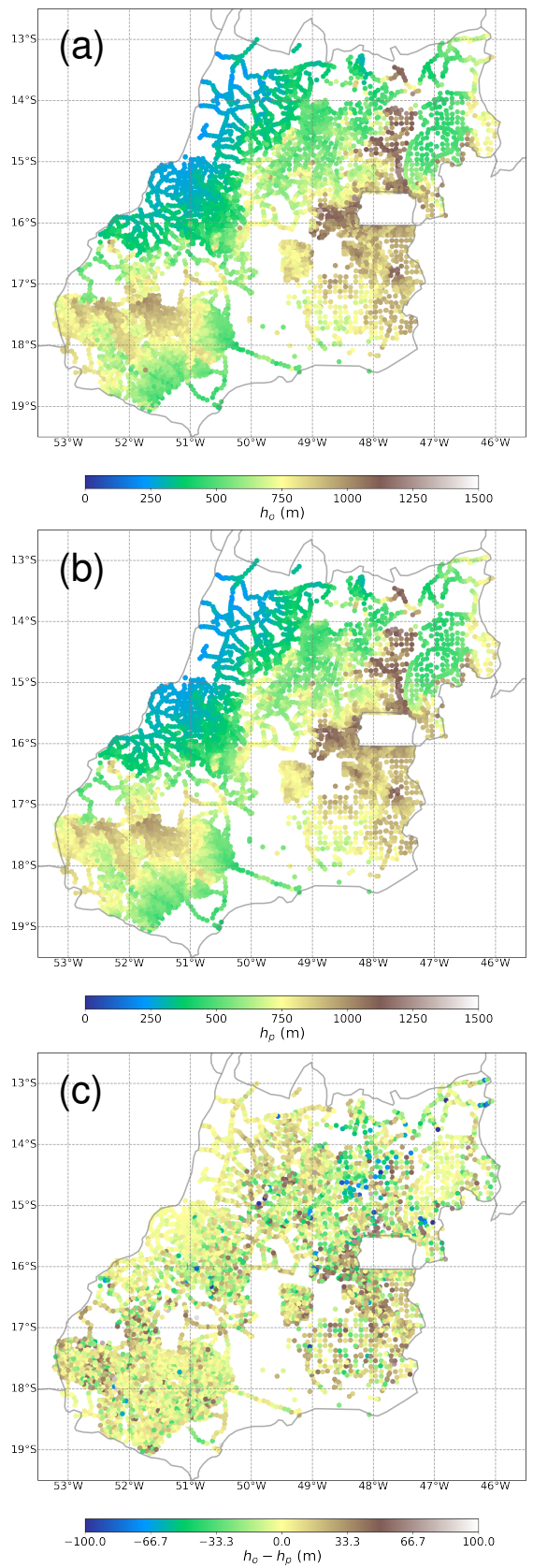

Figure 4: Geometric heights of Goiás state. (a) The True $h_{p}$, (b) the Modeled $h_{p}$ and (c) the Residual map.

To deeper the analysis a little more, we also provide a residuals histogram, as can be seen in Figure 5. It is highlighted that the mean of residuals is $1.97 \mathrm{mGal}$ with a standard deviation of $25.84 \mathrm{mGal}$. These values show that the modeled geometric height presents small discrepancies when compared to the observed heights.

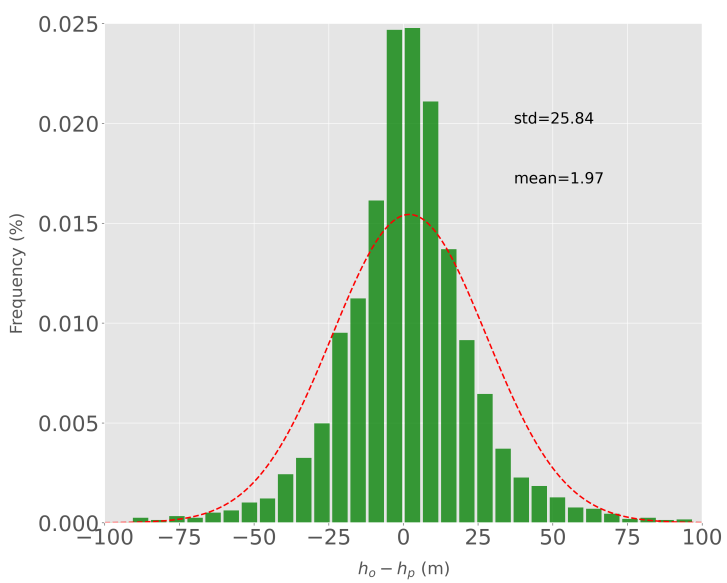

Figure 5: The residuals of geometric heights.

In order to bespeak the possible reason for such small differences, we also analyze a crossplot involving both observed and predicted geometric heights, as presented in Figure 6. The linear regression factor, for this example, is $R=0.99$, which indicates a very good linear relation between the two data-sets. A natural conclusion is that the modeled geometric heights computed by EIGEN-6C4 are acceptable for Goiás state. There is not over or under prediction of this data.

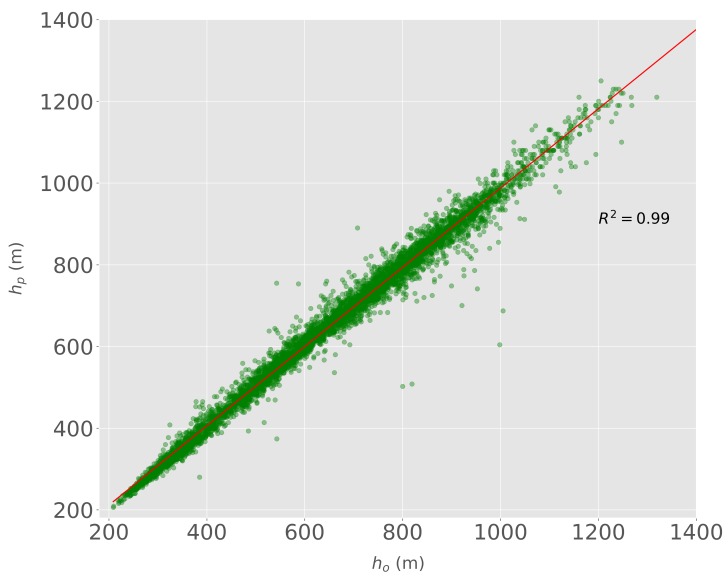

Figure 6: Crossplot of geometric heights of Goiás state.

\section{Gravity disturbances $(\delta \mathbf{g})$}

We are now ready to analyze the gravity disturbances of Goiás state. Figure 7 (a,b and c) shows the observed $\delta \mathbf{g}$, the modeled $\delta \mathbf{g}$ and the residuals map. Again, there are some relevant discrepancies in the residuals, especially at the central part of the area, which might be related to some bad geometric height estimates. 

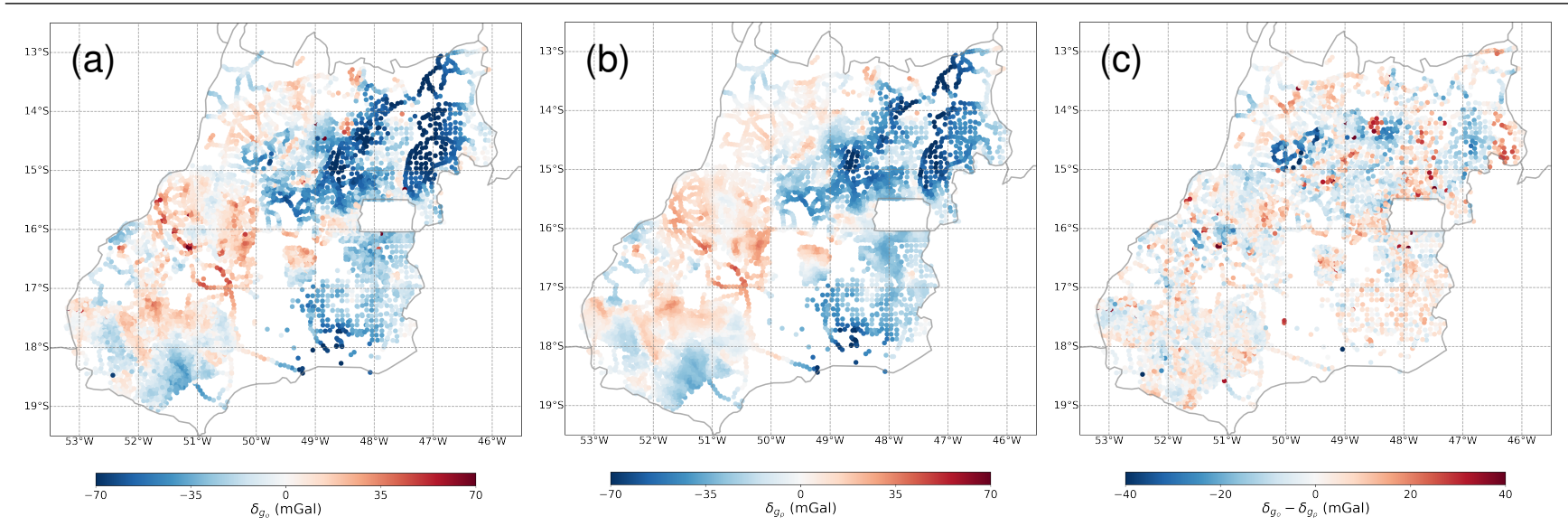

Figure 7: Gravity disturbances of Goiás state. (a) Observed $\delta g$, (b) Modeled $\delta g$ and (c) Residual map.

Further analysis are required to mitigate the potentialities and limitations of this approach. To do so, Figure 8 presents the residuals histogram of both observed and modeled $\delta \mathbf{g}$. Mean and standard deviation values (i.e., $-0.56 \mathrm{mGal}$ and $9.78 \mathrm{mGal}$ ) indicates a very accepted overall fit. Once again, there is no prominent tendency into the data-set modeled by EIGEN-6C4. However, the amplitudes ranges of the residuals larger than the previously results.

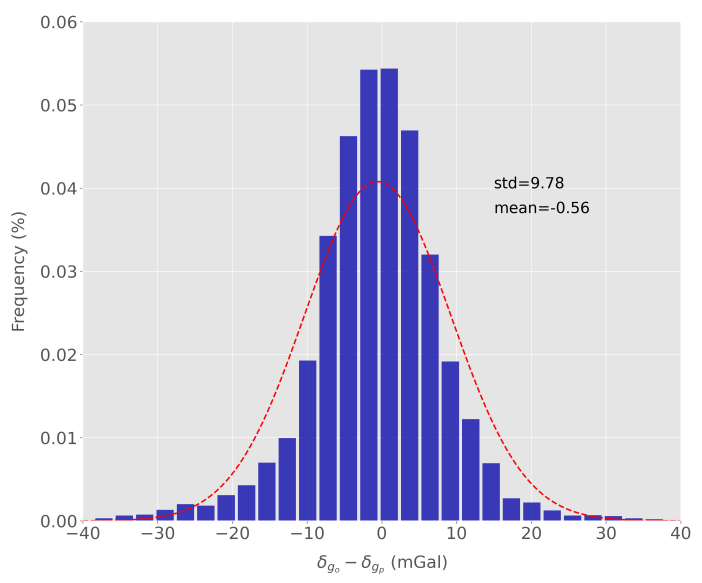

Figure 8: The histogram of gravity disturbance residuals.

Figure 9 shows the dispersion analysis for the gravity disturbances. We now observe that the data-set is way more disperse, which is corroborated by a linear regression coefficient of $R=0.84$. We can also see that very few data points are badly predicted. This aspect might be related to the limitations of the EIGEN-6c4 prediction of terrestrial gravity data.

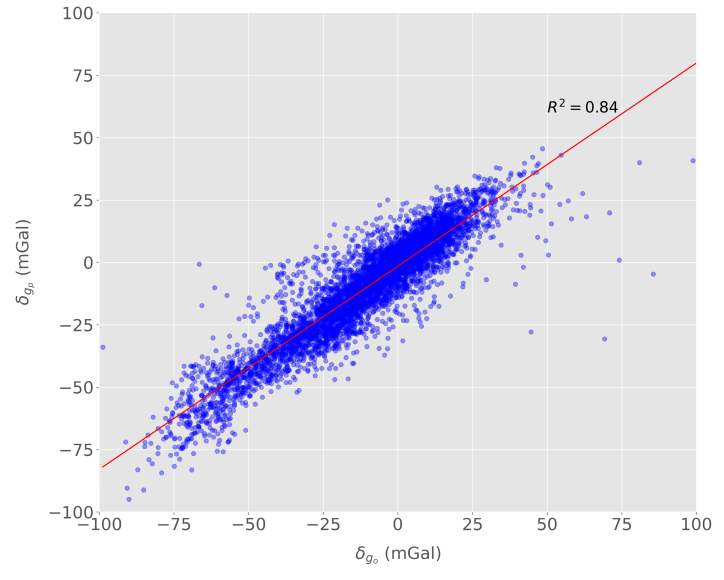

Figure 9: The crossplot of gravity disturbances datasets of Goiás state.

\section{Conclusions}

This work aims to present a comparative study for observed and predict gravity disturbances. Additionally, observed and predict geometrical heights are also considered in this work. Both data-sets comprise Goiás state, Midwest Brazil. With this, we could verify the predictability of Global Earth Model EIGEN 6c4 in more specific set of applications. We use ICGEM website to calculate the predict data-sets and compare these with the observed data, acquired in a set of land surveys. In a general view, the regional trends in both data-sets are represented by the model quite well. However, severe discrepancies in data amplitudes are noted, which is probably related to the truncation of the spherical harmonics expansion of $n=2190$. This study shows that Global Models are very efficient in predicting data with large wavelengths. Statistical analysis comprising histograms and crossplots of both gravity disturbances and geometrical heights endorses the presented discussion. As a future perpective, we could deal with a more regional gravity survey to verify if the modeled-data amplitudes are better represented. Additionally, other Global Earth Models could also be considered for a broader comparison and verification. 


\section{Acknowledgments}

Authors would like to thank Observatório Nacional and Universidade Federal Fluminense for providing all necessary technical resources to the development of this study.

\section{References}

Barbosa, D. K. S., 2017, Gravimetria por satélite aplicada à pesquisa mineral: estudo de casos no brasil.

Barthelmes, F., 2009, Definic̃ã dos funcionais do geopotencial e seu cálculo a partir de modelos harmônicos esféricos: http: // publicac̃ões. iasspotsdam. de / pubman / item / escidoc, 104132, 0902-2.

- 2014, Global models: Encyclopedia of Geodesy, Springer International Publishing, 1-9.

Bell, R. E., V. A. Childers, R. A. Arko, D. D. Blankenship, and J. M. Brozena, 1999, Airborne gravity and precise positioning for geologic applications: Journal of Geophysical Research: Solid Earth, 104, 15281-15292.

Escobar, I., 1987, A rede gravimétrica fundamental brasileira: Observátorio Nacional, Departamento de Geofísica, 2.

Escobar, I. P., 2000, Forma e dimensões da Terra: Universidade do Estado do Rio de Janeiro. Departamento de Engenharia Cartográfica.

F'orste, C., S. Bruinsma, O. Abrikosov, J. Lemoine, T. Schaller, H. Gtze, J. Ebbing, J. Marty, F. Flechtner, G. Balmino, and R. Biancale, 2014, Eigen-6c4 the latest combined global gravity field model including goce data up to degree and order 2190 of gfz potsdam and grgs toulouse: GFZ Data Services. doi, 10.

Förste, C., S. Bruinsma, O. Abrikosov, S. Rudenko, J.-M. Lemoine, J.-C. Marty, K. H. Neumayer, and R. Biancale, 2016, Eigen-6s4 a time-variable satellite-only gravity field model to $\mathrm{d} / \mathrm{o} 300$ based on lageos, grace and goce data from the collaboration of $\mathrm{gfz}$ potsdam and grgs toulouse: GFZ Data Services.

Förste, C., F. Flechtner, R. Schmidt, R. König, U. Meyer, R. Stubenvoll, M. Rothacher, F. Barthelmes, K. Neumayer, R. Biancale, et al., 2006, A mean global gravity field model from the combination of satellite mission and altimetry/gravimetry surface gravity data: Presented at the European Geosciences Union General Assembly 2006.

Hallam, K. A. T., 2019, Modelagem regional do campo de gravidade utilizando pontos de massa em coordenadas geodésicas: PhD thesis, Observatório Nacional.

Halpenny, J. F., and D. M. Darbha, 1995, Airborne gravity tests over lake ontario: Geophysics, 60, 61-65.

Hofmann-Wellenhof, Bernhard e Moritz, H., 2005, Fundamentals of potential theory: GeodÃ@sia FÃsica, 3-41.

Junior, C. A. C. e. C., G. d. N. Guimarães, and N. C. Ferreira, 2019, Evolução da infraestrutura gravimétrica no brasil: Geociências (São Paulo), 37, 361-384.

Li, X., and H.-J. Götze, 2001, Ellipsoid, geoid, gravity, geodesy, and geophysics: Geophysics, 66, 1660-1668.

Molina, E. C., 2001, Gravimetria: Fundamentos e aplicac̃ões - módulo 1.

Moritz, H., 1980, Advanced physical geodesy: Advances in Planetary Geology.

Schwintzer, P., and C. Reigber, 2002, The contribution of gps flight receivers to global gravity field recovery: Journal of Global Positioning Systems, 1, 61-63.
Torge, W., 1989, Gravimetry: de Gruyter. 